\title{
New Conditions for a Total Neutrino Conversion in a Medium
}

\author{
M. V. Chizhov ${ }^{1}$ and S. T. Petcov ${ }^{2,3}$ \\ ${ }^{1}$ Centre for Space Research and Technologies, Faculty of Physics, University of Sofia, 1164 Sofia, Bulgaria \\ ${ }^{2}$ Scuola Internazionale Superiore di Studi Avanzati, Istituto Nazionale di Fizica Nucleare, Sezione di Trieste, \\ I-34014, Trieste, Italy \\ ${ }^{3}$ Institute of Nuclear Research and Nuclear Energy, Bulgarian Academy of Sciences, 1784 Sofia, Bulgaria
}

\begin{abstract}
A new effect of total neutrino conversion is possible when neutrino propagates through multi-layer medium of nonperiodic constant density layers. The effect can take place in the oscillations in the Earth of the Earth-core-crossing solar and atmospheric neutrinos.
\end{abstract}

Neutrino oscillations were proposed by B. Pontecorvo [1] in analogy with the $K^{0}-\bar{K}^{0}$ oscillations (see also [2,3]). The pattern of oscillations of ultra-relativistic neutrinos in vacuum depends in the simplest case of twoneutrino mixing on the vacuum mixing angle $\theta$, the ratio of the neutrino squared mass difference and the neutrino energy, $\Delta m^{2} / E$, and the time or distance $t \cong X$ traveled by the neutrinos. The probability of transition between the weak-eigenstate neutrinos $\nu_{\alpha}$ and $\nu_{\beta}$ $(\alpha \neq \beta=e, \mu, \tau, s)$ in this case,

$$
P_{\alpha \beta}=\sin ^{2}(2 \theta) \sin ^{2} \phi,
$$

is determined by the angle $2 \theta$ and the phase $\phi=$ $\left(\Delta m^{2} / 4 E\right) X$. Therefore, the necessary condition for a total neutrino conversion is $\theta=\pi / 4$, while the external parameter $X$ can, in principle, be chosen to have $P_{\alpha \beta}=1$.

When neutrinos propagate in matter an additional phase difference can arise between the $\nu_{\alpha}$ and $\nu_{\beta}$ states due to the difference $V_{\alpha \beta}$ of their effective potentials in matter. In the case of matter with constant density and chemical composition, this difference can be accounted for through the mixing angle in matter, $\theta_{m}$, and the effective squared mass difference

$$
\Delta m_{\text {eff }}^{2}=\Delta m^{2} \sqrt{\left(\cos (2 \theta)-\frac{2 E V_{\alpha \beta}}{\Delta m^{2}}\right)^{2}+\sin ^{2}(2 \theta)},
$$

in such a way that the transition probability in matter,

$$
P_{\alpha \beta}=\sin ^{2}\left(2 \theta_{m}\right) \sin ^{2} \phi_{m},
$$

has the same form as that in vacuum, where $\phi_{m}=$ $\left(\Delta m_{\text {eff }}^{2} / 4 E\right) X$. The mixing angle in matter $\theta_{m}$ is given by the familiar resonance-type expression

$$
\sin ^{2}\left(2 \theta_{m}\right)=\frac{\sin ^{2}(2 \theta)}{\left(\cos (2 \theta)-\frac{2 E V_{\alpha \beta}}{\Delta m^{2}}\right)^{2}+\sin ^{2}(2 \theta)} .
$$

It can be maximal, $\theta_{m}=\pi / 4$, even in the case of a small vacuum mixing angle $\theta$, when the resonance condition

$$
\frac{\Delta m^{2}}{2 E} \cos (2 \theta)-V_{\alpha \beta}=0,
$$

is satisfied, where $V_{e \mu}=\sqrt{2} G_{F} N_{e}, V_{e s}=\sqrt{2} G_{F}\left(N_{e}-\right.$ $\left.\frac{1}{2} N_{n}\right)$, etc., $N_{e, n}$ being the electron and neutron number densities of matter. This is the well-known MSW effect 画.

The neutrino oscillations are a pure quantum mechanical phenomenon and it is interesting to investigate the interference effects in the neutrino transitions when neutrinos pass through a multi-layer medium of (nonperiodic) constant density layers. In this case the transition probability amplitude is a sum of products of the probability amplitudes of the transitions in each layer. In what follows we will show that due to the constructive interference between the different amplitudes, new solutions for a total neutrino conversion exist even when the resonance condition (3) is not fulfilled in any of the layers of the medium. This phenomenon differs from the parametric resonance effect possible in a medium with periodic density distribution [5]. In order for the new effect to take place, the passage of neutrinos through two layers of different constant density is sufficient. Moreover, the strong resonance-like enhancement of the probabilities of the $\nu_{2} \rightarrow \nu_{e}, \nu_{\mu} \rightarrow \nu_{e}\left(\nu_{e} \rightarrow \nu_{\mu(\tau)}\right), \nu_{e} \rightarrow \nu_{s}$, etc. transitions in the Earth of the Earth-core crossing solar and atmospheric neutrinos (see, e.g., [6 8] and the references quoted therein), is due to this effect. It was shown in [9] that the standard interpretation of this enhancement (see, e.g., [6.8]) as being due to the MSW effect in the Earth core is incorrect and it was suggested that the enhancement is generated by a new type of resonance - "neutrino oscillation length resonance" (NOLR). At small mixing angles and in the case of the transitions $\nu_{2} \rightarrow \nu_{e}$ and $\nu_{\mu} \rightarrow \nu_{e}$, for instance, the values of the parameters at which the maximal neutrino conversion takes place for neutrinos traversing the Earth core are rather close to the values of the parameters at which the NOLR can occur. In all transitions, however, only the maximal neutrino conversion mechanism is operative for the Earth-core-crossing neutrinos. 
Consider the simple case of media consisting of two layers having constant but different densities and chemical compositions. Then the mixing angles in matter for the first and the second layer, $\theta_{m}^{\prime}$ and $\theta_{m}^{\prime \prime}$, are constant. We shall assume without loss of generality that

$$
0 \leq V_{\alpha \beta}^{\prime}<V_{\alpha \beta}^{\prime \prime},
$$

where $V_{\alpha \beta}^{\prime}$ and $V_{\alpha \beta}^{\prime \prime}$ are the effective potential differences in the first and the second layer, respectively. Our analysis will apply to the case when the first layer is vacuum $V_{\alpha \beta}^{\prime}=0$ as well, because the neutrino oscillations in vacuum and in constant-density medium are described by the same equations (see (11) and (2)). The probability amplitude of the transition $\nu_{\alpha} \rightarrow \nu_{\beta}$ after neutrinos have crossed the two layers represents a sum of two terms:

$$
A_{\alpha \beta}=A_{\alpha \alpha}^{\prime} A_{\alpha \beta}^{\prime \prime}+A_{\alpha \beta}^{\prime} A_{\beta \beta}^{\prime \prime},
$$

where

$$
\begin{aligned}
& A_{\alpha \alpha}^{\prime}=\cos \phi_{m}^{\prime}+i \cos \left(2 \theta_{m}^{\prime}\right) \sin \phi_{m}^{\prime} \\
& A_{\alpha \beta}^{\prime \prime}=-i \sin \left(2 \theta_{m}^{\prime \prime}\right) \sin \phi_{m}^{\prime \prime} \\
& A_{\alpha \beta}^{\prime}=-i \sin \left(2 \theta_{m}^{\prime}\right) \sin \phi_{m}^{\prime} \\
& A_{\beta \beta}^{\prime \prime}=\cos \phi_{m}^{\prime \prime}-i \cos \left(2 \theta_{m}^{\prime \prime}\right) \sin \phi_{m}^{\prime \prime} .
\end{aligned}
$$

Here $A_{\alpha \alpha}^{\prime}, A_{\alpha \beta}^{\prime}$ and $A_{\alpha \beta}^{\prime \prime}, A_{\beta \beta}^{\prime \prime}$ are the probability amplitudes describing the oscillations in the first and in the second layer, respectively, $\phi_{m}^{\prime}=\left(\Delta m_{e f f}^{\prime 2} / 4 E\right) X^{\prime}$ and $\phi_{m}^{\prime \prime}=\left(\Delta m_{\text {eff }}^{\prime \prime 2} / 4 E\right) X^{\prime \prime}$ are half of the phase differences accumulated by the neutrino energy eigenstates in the first and in the second layer, and $X^{\prime}$ and $X^{\prime \prime}$ are the lengths of paths of the neutrinos in the corresponding layers. The transition probability

$$
\begin{aligned}
P_{\alpha \beta}=\left|A_{\alpha \beta}\right|^{2} & =\left|A_{\alpha \alpha}^{\prime}\right|^{2}\left|A_{\alpha \beta}^{\prime \prime}\right|^{2}+\left|A_{\alpha \beta}^{\prime}\right|^{2}\left|A_{\beta \beta}^{\prime \prime}\right|^{2} \\
& +2 \operatorname{Re}\left(\left(A_{\alpha \alpha}^{\prime} A_{\alpha \beta}^{\prime \prime}\right)^{*} A_{\alpha \beta}^{\prime} A_{\beta \beta}^{\prime \prime}\right)
\end{aligned}
$$

is a sum of two products of the probabilities of the neutrino oscillations in the different layers and of the interference term. The latter can play a crucial role in the enhancement of the neutrino transitions.

For monoenergetic neutrinos the path lengths $X^{\prime}$ and $X^{\prime \prime}$ can be considered as independent variables of the system, as long as the mixing angles in matter $\theta_{m}^{\prime}$ and $\theta_{m}^{\prime \prime}$ are fixed. Varying these variables we get a system of equations for the local maxima of $P_{\alpha \beta}$,

$$
\frac{\mathrm{d} P_{\alpha \beta}}{\mathrm{d} \phi_{m}^{\prime}}=0, \quad \frac{\mathrm{d} P_{\alpha \beta}}{\mathrm{d} \phi_{m}^{\prime \prime}}=0,
$$

provided the supplementary conditions on the values of the second derivatives of $P_{\alpha \beta}$ at the points where equations (8) hold,

$$
\frac{\mathrm{d}^{2} P_{\alpha \beta}}{\mathrm{d} \phi_{m}^{\prime 2}}<0, \quad \frac{\mathrm{d}^{2} P_{\alpha \beta}}{\mathrm{d} \phi_{m}^{\prime 2}} \frac{\mathrm{d}^{2} P_{\alpha \beta}}{\mathrm{d} \phi_{m}^{\prime \prime}{ }^{2}}-\left(\frac{\mathrm{d}^{2} P_{\alpha \beta}}{\mathrm{d} \phi_{m}^{\prime} \mathrm{d} \phi_{m}^{\prime \prime}}\right)^{2}>0,
$$

are fulfilled. This system has four types of different solutions determining the local and absolute maxima of the transition probability. The first one

$$
\text { solution } I:\left\{\begin{array}{l}
\cos \phi_{m}^{\prime}=0, \text { or } 2 \phi_{m}^{\prime}=\pi\left(2 k^{\prime}+1\right), \\
\sin \phi_{m}^{\prime \prime}=0, \text { or } 2 \phi_{m}^{\prime \prime}=2 \pi k^{\prime \prime},
\end{array}\right.
$$

where $k^{\prime}, k^{\prime \prime}=0,1, \ldots$, is valid in the region $I$ (Fig. 1)

$$
\text { region } I: \cos \left(2 \theta_{m}^{\prime}\right) \leq 0
$$

and corresponds to a neutrino conversion in the first layer only: $A_{\alpha \beta}^{\prime \prime}=0$. In this case the interference term in (7) is zero and the transition probability in the two layers is defined by the transition probability in the first layer

$$
\text { type I : } P_{\alpha \beta}^{\max }=\sin ^{2}\left(2 \theta_{m}^{\prime}\right) \text {. }
$$

A total neutrino conversion, $P_{\alpha \beta}=1$, occurs when the MSW resonance condition in the first layer,

$$
\cos \left(2 \theta_{m}^{\prime}\right)=0
$$

is satisfied. Thus, the absolute maxima in region $I$ can be ascribed to the MSW effect in the first layer.

The second solution,

$$
\text { solution II : }\left\{\begin{array}{l}
\sin \phi_{m}^{\prime}=0, \text { or } 2 \phi_{m}^{\prime}=2 \pi k^{\prime}, \\
\cos \phi_{m}^{\prime \prime}=0, \text { or } 2 \phi_{m}^{\prime \prime}=\pi\left(2 k^{\prime \prime}+1\right),
\end{array}\right.
$$

$k^{\prime}, k^{\prime \prime}=0,1, \ldots$, is realized in region $I I$ (Fig. 1),

$$
\text { region } I I: \cos \left(2 \theta_{m}^{\prime \prime}\right) \geq 0,
$$

and is completely analogous to the previous one, with the two layers interchanged. The phase requirements (14) reduce again the expression (7) to only one term

$$
\text { type II : } P_{\alpha \beta}^{\max }=\sin ^{2}\left(2 \theta_{m}^{\prime \prime}\right) \text {, }
$$

with no contribution from the interference term as $A_{\alpha \beta}^{\prime}=$ 0 . Thus, a total neutrino conversion in this case can be ascribed to the MSW effect in the second layer.

We get a very different mechanism of enhancement of the neutrino transition probability in the region

$$
\left\{\begin{array}{l}
\cos 2 \theta_{m}^{\prime}>0 \\
\cos 2 \theta_{m}^{\prime \prime}<0,
\end{array}\right.
$$

located between the regions $I$ and $I I$. The maxima of $P_{\alpha \beta}$ in this region are caused by maximal contribution of the interference term in equation (7) for $P_{\alpha \beta}$, taking place when the two vectors $\mathbf{z}_{1}=\left(\operatorname{Re}\left(A_{\alpha \alpha}^{\prime} A_{\alpha \beta}^{\prime \prime}\right), \operatorname{Im}\left(A_{\alpha \alpha}^{\prime} A_{\alpha \beta}^{\prime \prime}\right)\right)$ and $\mathbf{z}_{2}=\left(\operatorname{Re}\left(A_{\alpha \beta}^{\prime} A_{\beta \beta}^{\prime \prime}\right), \operatorname{Im}\left(A_{\alpha \beta}^{\prime} A_{\beta \beta}^{\prime \prime}\right)\right)$ in the complex plane are collinear and point in the same direction.

Indeed, the third solution, 


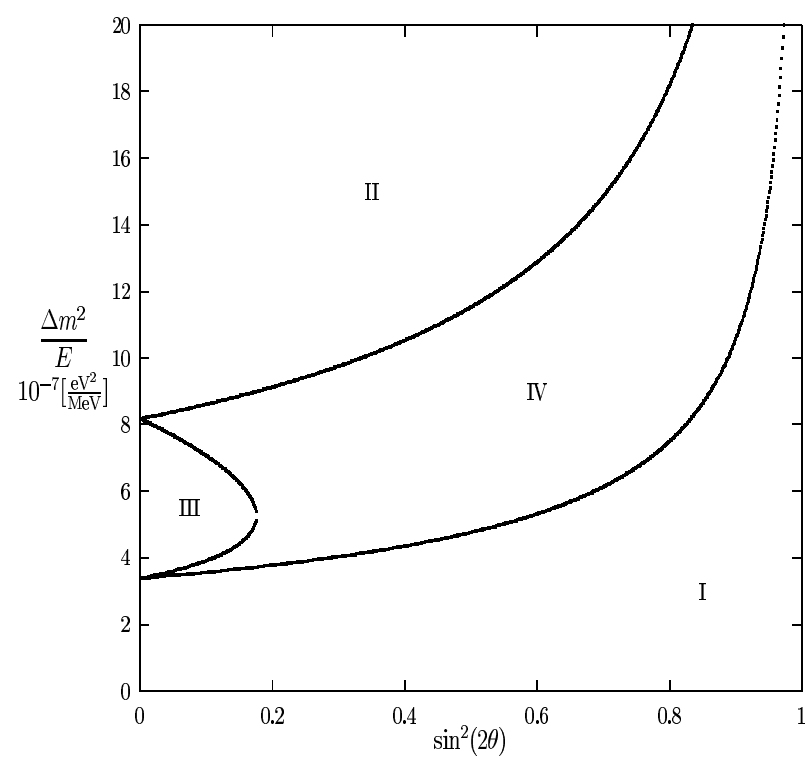

FIG. 1. The regions of the four different solutions for the maxima of the transition probability $P_{e \mu}=P_{\mu e}$ in a two-layer medium. The two different layers correspond to the mantle and the core of the Earth.

$$
\text { solution III : }\left\{\begin{array}{l}
\cos \phi_{m}^{\prime}=0, \text { or } 2 \phi_{m}^{\prime}=\pi\left(2 k^{\prime}+1\right) \\
\cos \phi_{m}^{\prime \prime}=0, \text { or } 2 \phi_{m}^{\prime \prime}=\pi(2 k+1)
\end{array}\right.
$$

where $k^{\prime}, k=0,1, \ldots$, leads to local maxima,

$$
\text { type } I I I: P_{\alpha \beta}^{\max }=\sin ^{2}\left(2 \theta_{m}^{\prime}-2 \theta_{m}^{\prime \prime}\right) \text {, }
$$

if the oscillation parameters belong to the finite region III (Fig. 1),

$$
\text { region } I I I: \cos \left(2 \theta_{m}^{\prime}-2 \theta_{m}^{\prime \prime}\right) \leq 0 .
$$

This maximum demonstrates the new effect of enhancement of the probability $P_{\alpha \beta}$, when the interference between the two terms in the r. h. side of (5) is maximal and constructive *. However, solution III does not provide an absolute maximum of $P_{\alpha \beta}$, except on the curve

$$
\cos \left(2 \theta_{m}^{\prime}-2 \theta_{m}^{\prime \prime}\right)=0 .
$$

Only the solutions of the fourth type,

solution $I V:\left\{\begin{array}{l}\tan \phi_{m}^{\prime}= \pm \sqrt{\frac{-\cos \left(2 \theta_{m}^{\prime \prime}\right)}{\cos \left(2 \theta_{m}^{\prime}\right) \cos \left(2 \theta_{m}^{\prime \prime}-2 \theta_{m}^{\prime}\right)}} \\ \tan \phi_{m}^{\prime \prime}= \pm \sqrt{\frac{-\cos \left(2 \theta_{m}^{\prime}\right)}{\cos \left(2 \theta_{m}^{\prime \prime}\right) \cos \left(2 \theta_{m}^{\prime \prime}-2 \theta_{m}^{\prime}\right)}}\end{array}\right.$

\footnotetext{
${ }^{*}$ The two-layer solutions (18) are the analogs of the solutions corresponding to the neutrino oscillation length resonance 9 in the case of the transitions in the Earth of the Earth-corecrossing neutrinos.
}

where the signs are correlated, provide an absolute maximum of $P_{\alpha \beta}$,

$$
\text { type } I V: P_{\alpha \beta}^{\max }=1,
$$

in the region $I V$ (Fig. 1),

$$
\text { region } I V:\left\{\begin{array}{l}
\cos 2 \theta_{m}^{\prime} \geq 0, \\
\cos 2 \theta_{m}^{\prime \prime} \leq 0, \\
\cos \left(2 \theta_{m}^{\prime \prime}-2 \theta_{m}^{\prime}\right) \geq 0 .
\end{array}\right.
$$

Note that these solutions do not imply any additional constraints on the mixing angles in matter of the type of the resonance conditions (13) or (21). In contrast to the case of the MSW effect, in which a total neutrino conversion can be realized only on the curve (3) in the $\sin ^{2}(2 \theta)-\Delta m^{2} / E$ plane of parameters, the new effect is possible in the whole 2-dimensional region $I V$.

When the widths of the layers $X^{\prime}$ and $X^{\prime \prime}$ are fixed, the neutrino energy $E$ is the only physical parameter which can be varied. The extrema condition reads in this case:

$$
\begin{aligned}
\frac{\mathrm{d} P_{\alpha \beta}}{\mathrm{d} E} & =\frac{\mathrm{d} P_{\alpha \beta}}{\mathrm{d} \phi_{m}^{\prime}} \frac{\mathrm{d} \phi_{m}^{\prime}}{\mathrm{d} E}+\frac{4 V_{\alpha \beta}^{\prime}}{\Delta m_{e f f}^{\prime 2}} \sin \left(2 \theta_{m}^{\prime}\right) \\
& \times \cos \left(2 \theta_{m}^{\prime}-2 \theta_{m}^{\prime \prime}\right) \sin \phi_{m}^{\prime} \cos \phi_{m}^{\prime \prime} \\
& \times\left\{\left[\frac{\cos \left(2 \theta_{m}^{\prime}\right)}{\cos \left(2 \theta_{m}^{\prime \prime}\right) \cos \left(2 \theta_{m}^{\prime}-2 \theta_{m}^{\prime \prime}\right)}+\tan ^{2} \phi_{m}^{\prime \prime}\right]\right. \\
& \times \sin \left(2 \theta_{m}^{\prime}\right) \cos \left(2 \theta_{m}^{\prime \prime}\right) \sin \phi_{m}^{\prime} \cos \phi_{m}^{\prime \prime} \\
& +\cos \left(2 \theta_{m}^{\prime}\right) \sin \left(2 \theta_{m}^{\prime \prime}\right) \cos \phi_{m}^{\prime} \sin \phi_{m}^{\prime \prime} \\
& \left.\times\left[\frac{1}{\cos \left(2 \theta_{m}^{\prime}-2 \theta_{m}^{\prime \prime}\right)}-\tan \phi_{m}^{\prime} \tan \phi_{m}^{\prime \prime}\right]\right\} \\
& +\left({ }^{\prime} \leftrightarrow{ }^{\prime \prime}\right)=0 .
\end{aligned}
$$

It is clear, that, in general, the solutions of the system (8) do not correspond to extrema in the variable $E$. Only the solutions (22) for the absolute maxima, which correspond to a total neutrino conversion, give the absolute maxima in any variable. The solutions (10), (14), and (18) for the local maxima with $P_{\alpha \beta}<1$ no longer correspond to extrema in the variable $E$. New solutions are possible, which do not coincide with the solutions corresponding to phases equal to multiples of $\pi$. As can be shown, the same conclusion is valid also if the width of one of the layers is determined by the width of the second layer. The indicated possibilities are realized, e.g., for neutrinos born in the Earth central region.

In the case of the solar and atmospheric neutrinos passing through the Earth core on the way to the detectors, the Earth density distribution can be approximated by a two-layer density profile, core - mantle, and neutrinos cross three layers: mantle - core - mantle. For the calculation of the probabilities of interest the two-layer model of the Earth provides a very good approximation to the more complicated density distributions predicted by the existing models of the Earth (see, e.g., [9 11]). Because of the spherical symmetry of the Earth, the lengths $X^{\prime}$ and 
$X^{\prime \prime}$ are not independent variables. However, as is shown in 12], the analogs of the solutions $I V$, corresponding to a total neutrino conversion, $P_{\alpha \beta}=1$, exist for the Earth

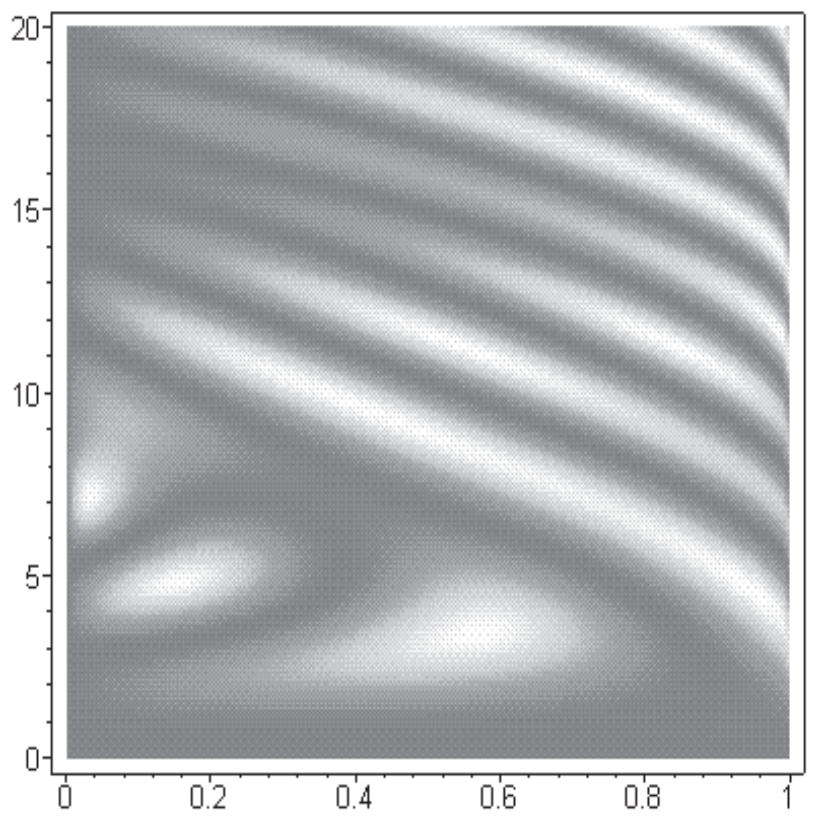

FIG. 2. The probability $P_{e \mu}=P_{\mu e}$ for the Earth center crossing neutrinos, as a function of $\sin ^{2} 2 \theta$ (horizontal axis) and $\Delta m^{2} / E\left[10^{-7} \mathrm{eV}^{2} / \mathrm{MeV}\right]$ (vertical axis). The greyscales correspond to different values of $P_{e \mu}: 0.0-0.10$ is represented by black, etc., while the white represents values in the interval 0.9 - 1.0. The points of total neutrino conversion (in the white regions), $P_{e \mu}=1$, correspond to solutions $I V$ for the Earth-core-crossing neutrinos, eq. (26).

density profile as well. For the $\nu_{\mu(e)} \rightarrow \nu_{e(\mu ; \tau)}, \nu_{e} \rightarrow \nu_{s}$ and $\bar{\nu}_{\mu} \rightarrow \bar{\nu}_{s}$ transitions $\left(\Delta m^{2} \cos 2 \theta>0\right)$ they read:

$$
\begin{aligned}
\tan \phi_{m}^{\prime} & = \pm \sqrt{\frac{-\cos 2 \theta_{m}^{\prime \prime}}{\cos \left(2 \theta_{m}^{\prime \prime}-4 \theta_{m}^{\prime}\right)}}, \\
\tan \phi_{m}^{\prime \prime} & = \pm \frac{\cos 2 \theta_{m}^{\prime}}{\sqrt{-\cos \left(2 \theta_{m}^{\prime \prime}\right) \cos \left(2 \theta_{m}^{\prime \prime}-4 \theta_{m}^{\prime}\right)}},
\end{aligned}
$$

where the signs are correlated; the solutions for the $\nu_{2} \rightarrow \nu_{e}$ transitions can formally be obtained from eq. (26) by replacing $2 \theta_{m}^{\prime \prime}$ and $2 \theta_{m}^{\prime}$ in the expressions for $\phi_{m}^{\prime}$ and $\phi_{m}^{\prime \prime}$ with $2 \theta_{m}^{\prime \prime}-\theta$ and $2 \theta_{m}^{\prime}-\theta$. Moreover, these solutions are responsible for the strong resonance-like enhancement of the $\nu_{2} \rightarrow \nu_{e}, \nu_{\mu} \rightarrow \nu_{e}, \nu_{e} \rightarrow \nu_{\mu(\tau)}, \nu_{e} \rightarrow \nu_{s}$, etc. transitions in the Earth of the Earth-core crossing solar and atmospheric neutrinos, discussed in [9] (see also [13]). In [9] this enhancement was interpreted to be due to the neutrino oscillation length resonance (NOLR) the analog of the solution (18) in the case of the Earthcore-crossing neutrinos. On the boder line between the regions where the NOLR and the maximal conversion solution (26) can be realized, the two solutions coincide and we have $P_{\alpha \beta}^{\max }=1$ at the points where they hold. However, the NOLR does not describe the local maxima, $P_{\alpha \beta}^{\max }<1$, in the variable $\Delta m^{2} / E$ at fixed $\sin ^{2} 2 \theta$ and/or fixed $h$ (for further details see [12]). In all transitions of interest only the maximal neutrino conversion mechanism is operative for the Earth-core-crossing neutrinos, both at small and large values of $\sin ^{2} 2 \theta$ (Fig. 2).

Acknowledgements. This work was supported in part by the Italian MURST under the program "Fisica Teorica delle Interazioni Fondamentali"; the work of S.T.P. was partially supported also by Grant PH-510 from the Bulgarian Science Foundation.

[1] B. Pontecorvo, Zh. Eksp. Teor. Fiz. 33, 549 (1957); ibid. 34, 247 (1958).

[2] Z. Maki, M. Nakagawa and S. Sakata, Prog. Theor. Phys. 28, 870 (1962).

[3] B. Pontecorvo, Zh. Eksp. Teor. Fiz. 53, 1717 (1967); V. Gibov and B. Pontecorvo, Phys. Lett. B28, 493 (1969). S.M. Bilenky and B. Pontecorvo, Phys. Rep. 41, 225 (1978).

[4] S.P. Mikheyev and A.Yu. Smirnov, Sov. J. Nucl. Phys. 42, 913 (1985); L. Wolfenstein, Phys. Rev. D17,2369 (1978).

[5] V.K. Ermilova et al., Short Notices of the Lebedev Institute 5, 26 (1986); E. Akhmedov, Yad. Fiz. 47, 475 (1988); P. Krastev and A.Yu. Smirnov, Phys. Lett. B226, 341 (1989).

[6] M. Cribier et al., Phys. Lett. B182, 89 (1986); A.J. Baltz and J. Weneser, Phys. Rev. D35, 528 (1987), D50, 5971 (1994); J.M. LoSecco, Phys. Rev. D47, 2032 (1993); J.M. Gelb, W. Kwong and S.P. Rosen, Phys. Rev. Lett. 78, 2296 (1997).

[7] M. Maris and S.T. Petcov, Phys. Rev. D 56, 7444 (1997).

[8] S.P. Mikheyev and A.Yu. Smirnov, in Massive Neutrinos in Astrophysics and in Particle Physics, Proceedings of the Moriond Workshop 1986, Tignes, France, (eds. O. Fackler and J. Tran Thanh Van, Editions Frontières, Gifsur-Yvette, 1986), p. 355; E.D. Carlson, Phys. Rev. D34, 1454 (1986); A. Dar et al., Phys. Rev. D35, 3607 (1987).

[9] S.T. Petcov, Phys. Lett. B434, 321 (1998), (E) B444, 584 (1998).

[10] M. Maris and S.T. Petcov, to be published.

[11] P.I. Krastev and S.T. Petcov, Phys. Lett. B205, 84 (1988).

[12] M. V. Chizhov and S. T. Petcov, Report SISSA 28/99/EP, March 1999 (hep-ph/9903424).

[13] M. Chizhov, M. Maris and S.T. Petcov, Report SISSA 53/98/EP, 31 July 1998 (hep-ph/9810501). 